\title{
Ion induced field effect in silicon in nematic liquid crystal cell
}

\author{
M.I. Gritsenko, S.I. Kucheev \\ Physics department, Chernigov pedagogical university, 53 G. Polubotka str., 14038 Chernigov, Ukraine \\ E-mail:kucheev@cn.relc.com
}

\begin{abstract}
An influence of ion charge on surface conductivity of silicon in $\operatorname{In}_{2} \mathrm{O}_{3} / 5 \mathrm{CB} /$ monocrystal silicon/Al structure, in which a specific resistance of silicon is much less than a specific resistance of liquid crystal layer, was studied. Within the frequency range of $10^{3}-10^{6} \mathrm{~Hz}$ of external voltage, a depletion state in silicon has been observed. We found that the field effect in silicon is induced by ion charges localized near silicon surface. It is shown that method based on visualization of $n^{+}$pockets on $n$-Si substrate by liquid crystal enables to estimate the impurity ion density near silicon surface. Analysis of the field effect induced by polarized charges formed by the prior action of DC voltage has allowed us to conclude that: 1) up to several volts of DC, a charge injection from silicon surface into liquid crystal is absent; 2) ion charge accumulated near silicon surface is partially adsorbed on silicon surface; 3) high voltage with "+" U polarity on silicon causes full absence of photosensitivity. We demonstrated that such type of structure could be used as liquid crystal spatial light modulator.
\end{abstract}

Keywords: liquid crystal, silicon, spatial light modulator, nondestructive testing.

Paper received 17.01.03; accepted for publication 16.06.03.

\section{Introduction}

It is well known [1] that an external electric field can modulate the surface conductivity of semiconductor (the so-called "field effect"). Usually, the field effect is induced by electric field of charged metal flat electrode placed near the semiconductor surface. However, if the metal electrode is not isolated by a dielectric film, generally more than $\sim 0.1 \mu \mathrm{m}$ thick, the realization of the field effect is very difficult because charges transferred through semiconductor surface prevent formation of an inversion layer in semiconductor. It is obvious that the ITO/ nematic/silicon type of structure in which dielectric film is absent and the specific resistance of silicon $\sim 5 \mathrm{Ohm} \cdot \mathrm{cm}$ (that is much less than the specific resistance of liquid crystal layer) the sufficiently strong transfer of charges is possible. As a result, at a first glance, it is very difficult to expect any surface modulation of conductivity in such structures. However, the recent results [2-5] show that such a type of structures can exhibit photosensitivity: under the action of light in the structure the reorientation of liquid crystal takes place in accordance with the light intensity distribution. It means that in such structures there is a high resistivity state of silicon.
The purpose of the present work is to demonstrate that a field effect in the silicon substrate in nematic cell is induced by ion charges accumulated near silicon surface.

\section{Experiment and discussion}

In our experiments we used the special silicon-liquid crystal structure in which the electrooptic effects are directly connected with the location of depletion layer boundary in silicon, Fig. 1. A number of high doped regions (as long pockets in silicon sample with equilibrium carrier density $n^{+} \sim 10^{19} \mathrm{~cm}^{-3}$ and $4 \mu \mathrm{m}$ width, $\sim 0.2 \mu \mathrm{m}$ depth $\mathrm{H}$ ) were formed in the $n$-type conductivity silicon sample with $n \sim 10^{15} \mathrm{~cm}^{-3}$ by ion doping. The distance between pockets was $\mathrm{W} \sim 20 \mu \mathrm{m}$. Aluminum layer from rear side forms the electrical contact to the silicon sample. We used liquid crystal $5 \mathrm{CB}$, which filled in the capillary between silicon surface and $\operatorname{In}_{2} \mathrm{O}_{3}$. Liquid crystal had planar orientation due to rubbed polyimide on $\operatorname{In}_{2} \mathrm{O}_{3}$ surface. The silicon surface has been gently rubbed to create the preferred direction of nematic. Note that optically one cannot see $n^{+}$pockets on silicon surface (as well as in assembled structure) because the reflectivity from silicon surface does not depend on the value of doping. 




Fig. 1. Schematic view of the experimental structure.

When an AC voltage is first time applied to a structure, the image that reproduces the $n^{+}$pocket location appears within 3-5 seconds (Fig. 2). Obviously, such a visualization can take place only if the resistance of $n$ silicon is comparable to that of the liquid crystal layer. Since $n^{+}>>n$ for silicon within pockets, we may neglect the depletion of $n^{+}$pockets, therefore, all the external voltage is applied to liquid crystal layer here. On the other hand, the surface of the non-doped $n$-silicon is depleted and its resistance becomes high. Hence, some part of the external voltage is dropped in the depleted layer. Therefore, Freedericks' transition starts over $n^{+}$pockets at lower voltages than over non-doped $n$-Si.

We studied the volt-ampere characteristics of such a structure under the constant voltage. It was stated that the branches of these characteristics were asymmetrical and the current through structure at the same voltages but different polarities are always larger by polarity "+" $\mathrm{U}$ on $\mathrm{Al}$ substrate. The asymmetry of the branches is

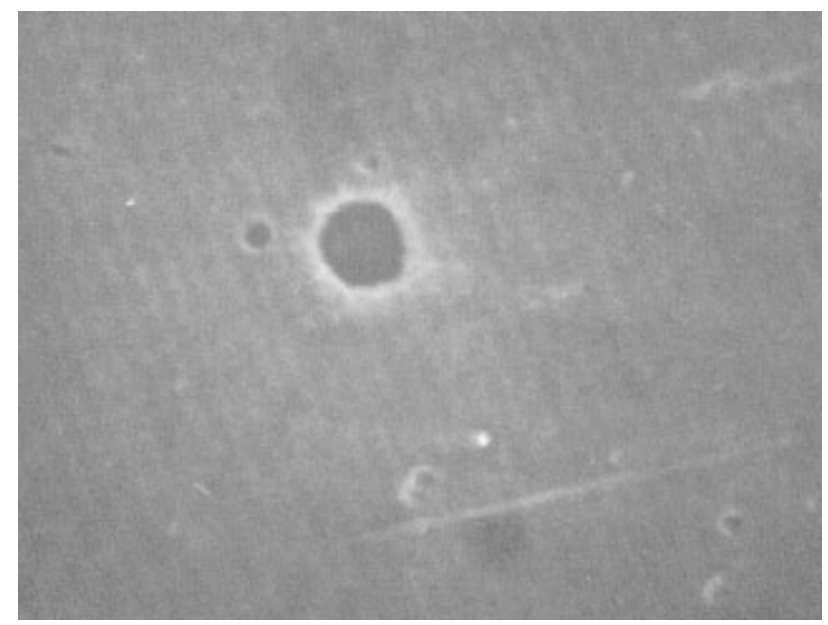

Fig. 2. Visualization of $n^{+}$pockets (white strips) on $n$-Si surface in ITO/5CB/Si/Al structure. Frequency $10^{5} \mathrm{~Hz}$. Voltage $4.2 \mathrm{~V}$. caused by the Shottky barrier in Si-Al interface of value of $\sim 0.7 \mathrm{~V}[1]$. Therefore, when AC voltage is applied to this structure, partial rectification takes place, and so the negative ion charge is accumulated near the silicon surface. When we use the silicon sample with ohmic contact to $\mathrm{Al}$ substrate, above mentioned visualization of $n^{+}$ pockets is absent. It means that the accumulated negative charge near silicon surface is also absent in this case.

Fig. 3 shows the dependence of the $n^{+}$pocket visualization threshold on the frequency of voltage and liquid crystal thickness. As seen from this figure, the visualization threshold does not depend on liquid crystal thickness. It means that silicon surface is depleted by electrical field of ions that are localized near silicon surface. We believe that the increase of threshold at low frequencies $\left(<10^{3} \mathrm{~Hz}\right)$ is due to current leakage through silicon/ liquid crystal interface, which prevents the formation of the depletion layer in silicon. When frequency is higher than $10^{3} \mathrm{~Hz}$, there is silicon surface depletion. It means that the charge transfer through silicon-liquid crystal interface is not so large. The increasing of threshold voltage at high frequencies $\left(>10^{5} \mathrm{~Hz}\right)$ is explained by the total decrease of the voltage drop on the cell as it takes place on a capacitor including both the liquid crystal and silicon layers, respectively.

We also found that if an external AC voltage is interrupted for a certain time $\Delta \mathrm{T}$ the structure returns to its initial state, when interruption time is longer than

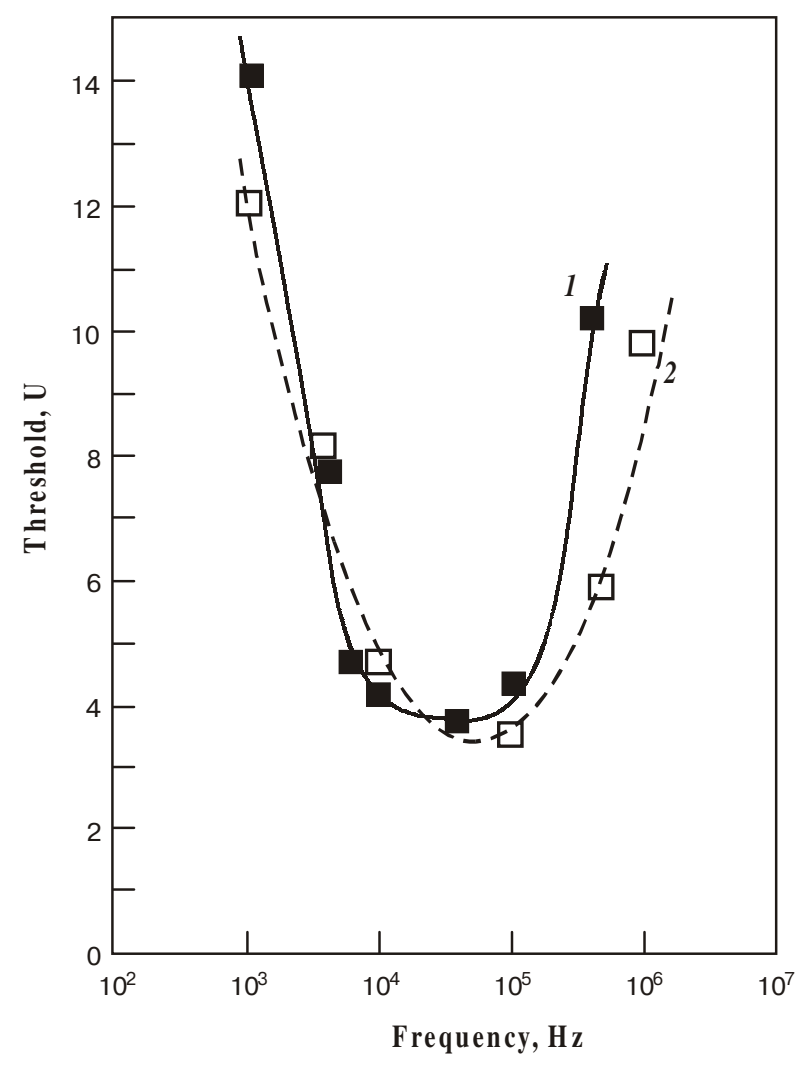

Fig. 3. Frequency dependence of threshold of $n+$ pockets visualization. Cell thicknesses are $1-20 \mu \mathrm{m}$ and $2-0.6 \mu \mathrm{m}$, respectively.

SQO, 6(2), 2003 
$10^{3}$ seconds. It is well known [6] that the dielectric relaxation time of the bulk charge is defined as $\tau_{D}=\varepsilon_{L} \varepsilon_{0} / \Omega$, where $\Omega$ and $\varepsilon_{L}$ are the conductivity and dielectric permitivity of the liquid crystal, respectively. For typical values of conductivity $\Omega=10^{-8}-10^{-10}(\mathrm{Ohm} \times \mathrm{cm})^{-1}, \tau_{D}$ is about some milliseconds [7]. Since $\Delta T \gg>\tau_{D}$, it may mean that negative charges accumulated near silicon surface are partially adsorbed by the surface.

The following phenomenon indirectly indicates adsorption significance. Some times, there are some accidentally placed round regions (for example, black round region shown in the center of Fig. 2) that repeat dried solvent microblobs appeared after silicon surface cleaning process. We believe that within these round places of cell $n^{+}$pockets are not visualized because depleted layer is not formed here. The impurity adsorbed on silicon surface plays the role of thin dielectric film, which blocks the influence of ion electric field on the silicon surface.

It is well known that the polarization of liquid crystals under DC voltage happens due to travelling impurity ions: positive ions move to cathode, negative ones move to anode. Let us consider how the high impurity ion density near the silicon surface influences on the electrooptical reaction of nematic liquid crystal in such a type of structures.

Let us switch on the DC voltage of polarity "_-"U on Al for time $t_{a c t}$, and then switch on a testing AC voltage. We found that the $n^{+}$pockets visualization starts by the time $t_{v i z}$ later, Fig. 4 . This time depends on the value of applied DC voltage and its duration. During the time $t_{v i z}$, there is no depleted state of the silicon surface, i.e. $\chi$ is close to zero. The absence of depletion in silicon is simply checked with a local excitation of the structure by He-Ne laser emission $(0.63 \mu \mathrm{m}, 2 \mathrm{~mW})$. Within this time delay $t_{v i z}$, there is no any reaction of liquid crystal in structure on the laser emission. So, we may conclude that when DC voltage of polarity "_" $\mathrm{U}$ on Al is applied, the positive ions are accumulated near silicon surface due to polarization. These ion charges attract electrons from the bulk of silicon toward its surface. Therefore, the depletion layer in silicon is not formed.

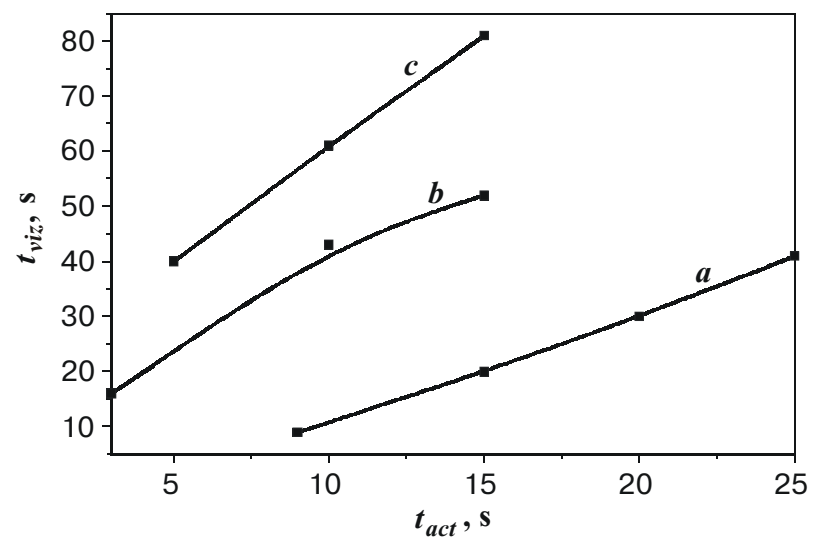

Fig. 4. Time $\left(t_{v i z}\right)$ of $n+$ pocket image appearance as a function of prior acting de voltage duration $\left(t_{a c t}\right): a-1, b-3, c-5$ volt. Testing voltage is $4.5 \mathrm{~V}$ and its frequency is $10^{5} \mathrm{~Hz}$, respectively.

$S Q O, 6(2), 2003$
Note the transition to $n^{+}$visualization state (and to photosensitive state too) at time $t_{v i z}$ does not happen simultaneously over all silicon surface but starts in the vicinity of defects of polyimide film on ITO. Further, the photosensitive area extends to being scoped all silicon surface. We believe that the conductivity of the structure in the vicinity of defects of polyimide film is larger than in neighboring defectless areas. Therefore, negative charge accumulation due to AC rectification and positive charge neutralization processes go more intensively here. Optically, the boundary between the photosensitive and non-photosensitive areas has well contrast, and its movement along the silicon surface is well observed.

Taking into account that carriers in nematic are negative ions, we can also conclude that charge injection of negative ions is absent up to several volts of DC voltage applied to the nematic cell. The large value of image appearance time $\tau_{v i z}$ in comparison with $\tau_{D}$ means that some part of this positive charge was also adsorbed at the silicon surface.

Let us consider the case when prior DC voltage of polarity "+"U on Si acted on the structure. In this case, the negative ions are accumulated in the liquid crystal near the silicon surface. As a result, the electric field of negative ions repulses the surface electrons into the bulk of silicon, and the inversion layer in silicon forms. However, when we apply AC voltage, $n^{+}$pockets are not visualized at the initial moment. It means that width $\chi$ of depletion layer in silicon is larger than $H$ (see Fig. 1). The evidence that there is silicon depletion is a light sensitivity of the structure under laser emission: there occurs a re-orientation of nematic liquid crystal within the spot on silicon illuminated by $\mathrm{He}-\mathrm{Ne}$ laser.

Fig. 5 gives the typical time dependencies of $n^{+}$pocket visualization versus prior DC voltage action. Initial disappearing of $n^{+}$pockets is explained that under the action of accumulated negative charge the width $\chi$ becomes larger than $H$. When we switch on the testing AC voltage, this negative charge starts to relax. Therefore, the boundary $\chi$ lifts and when $\chi \sim H n^{+}$pockets start to be visualized. Visualization time $T_{a p p}$ is proportional to duration of DC voltage action only for short periods of time and no high voltages (Fig. 5, up to arrow on 1st curve). It means that at this condition accumulated charge depletes a silicon only and does not cause other effects. However, if voltage is higher or its duration is longer, the visualization time $T_{a p p}$ strong decreases, i.e. the cell restores to initial state more quickly, Fig. 5 (curves 2, 3). Moreover, we found that strong voltage action approximately equal $10 \mathrm{~V}$ and several seconds duration causes full disappearing of photosensitivity of structure. Note that structure restores into initial state for several hours in this case. We believe that under the action of high voltages with "+" polarity on silicon the processes connected with silicon surface modification start to be realized. For example, the processes of etching or oxidation of $\mathrm{SiO}_{2}$ or $\mathrm{Si}$ surface can play an important role.

Exactly known width $\chi$ when it sets equal to depth $H$, gives us a possibility to estimate the surface charge density $\sigma$ by the following way. 


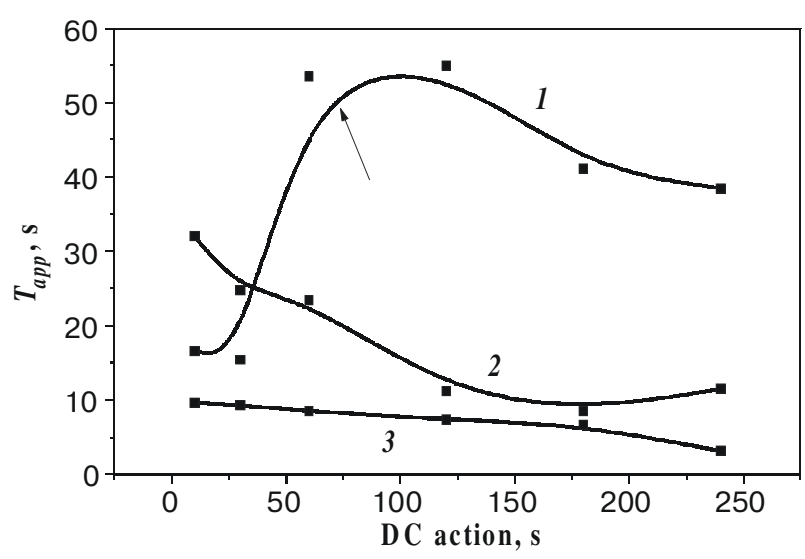

Fig. 5. Dependence of $n^{+}$-pocket visualization time, $T_{a p p}$, versus duration of preliminary DC voltage action. Testing AC voltage: $10^{5} \mathrm{~Hz}, 4.3 \mathrm{~V}$. The $1,2,3$ curves correspond to the "-" DC voltages 1,3 and $5 \mathrm{~V}$, respectively.

If current leakage through $\mathrm{Si} / \mathrm{LC}$ is negligibly small, we may use the following formula for determining the depleted layer width $\chi$, at high frequencies of the applied voltage, valid for classical Metal/Oxide/Semiconductor (MOS) structures [1].

$\chi=\left(\varepsilon \varepsilon_{0}\left(-2\left|\phi_{n}\right|+U\right) / \varepsilon N_{d}\right)^{1 / 2}$

where $\varepsilon$ is the dielectric permitivity of silicon, $\varepsilon_{0}$ is a dielectric constant, $U$ is voltage applied to semiconductor, $N_{d}$ is donor density in silicon. The surface potential is $\phi_{n}=(k T / e) \ln \left(N_{d} / n_{i}\right)$, where $n_{i}$ is the intrinsic carrier density in silicon $[1]\left(\sim 10^{10} \mathrm{~cm}^{-3}\right)$. In our case, $N_{d} \sim 10^{19} \mathrm{~cm}^{-3}$, therefore $\phi_{n}$ has the value $\sim 0.3 \mathrm{~V}$.

The charged layer of ions with density $\sigma=q N_{s}$, where $q$ is an ion charge, $N_{s}$ is a surface ion density induces the surface electric field $E_{S}[8,9]$ on silicon surface. An electric potential $\phi(z)$ obeys one-dimension Poisson's equation:

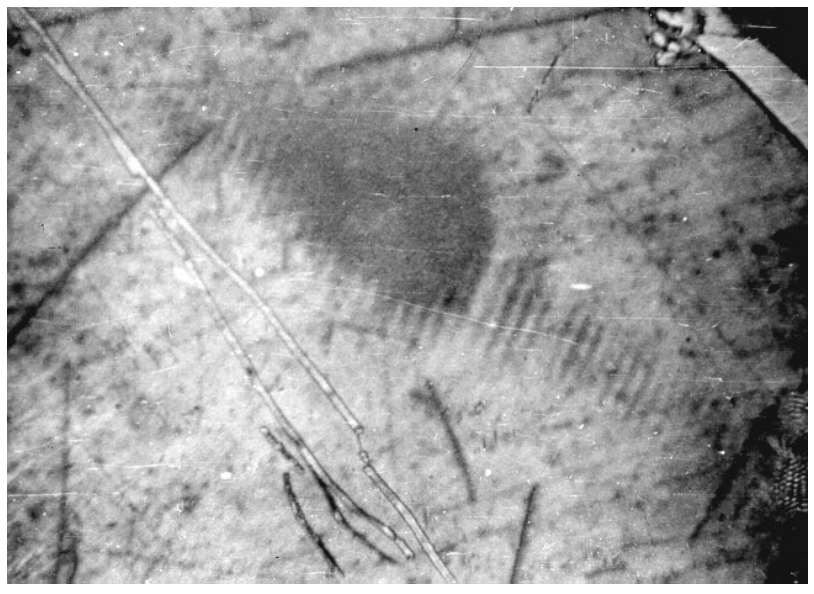

$a$ $d^{2} \phi(z) / d z^{2}=-\phi(z) / L^{2} D$

where $L_{D}=\left(\varepsilon \varepsilon_{0} k T / 2 n e^{2}\right)^{1 / 2}$ is the Debye length for silicon (in our case $L_{D} \sim 0.1 \mu \mathrm{m}$ ). The solution of the equation (2) is $\phi(z)=A \exp \left(-z / L_{D}\right)$. Using border conditions $E_{S}=\sigma / 2 \varepsilon \varepsilon_{0}$ and taking into account that on silicon surface $E_{s}=$ $=-d \phi(0) / d Z=A / L_{D}$ we obtain $A=\sigma L_{D} / \varepsilon \varepsilon_{0}$. The value of A (in volts) amounts to $\sim N_{S} \times 1.6 \times 10^{-12}$ [where $N_{S}$ is in $\left.1 / \mathrm{cm}^{2}\right]$. So, substituting $\mathrm{U}=\mathrm{A}$ into (1), we get that for the formation of a depleted layer with the width $\chi$ equal to $n^{+}$-pockets depth $H \sim 0.2 \mu \mathrm{m}$ the surface ion density $N_{s} \sim 10^{12} 1 / \mathrm{cm}^{2}$ is required. The calculated value of $N_{s}$ is in a good agreement with the estimation of surface ion density obtained in Ref.[10] through other approaches.

The strong dependence of depleted layer width on the parameters of external voltage is confirmed by the following experiments. We used the silicon substrate as shown in Fig. 1 but without $n^{+}$pockets. When we applied AC voltage, such structure becomes photosensitive, Fig. 6a. It means that silicon surface is depleted, and such structure can operate as spatial light modulator. Using a diffracted He-Ne laser beam, we obtained the resolution more than about 40 lines $/ \mathrm{mm}$. If we switch on additionally DC voltage with "+"U polarity on Al substrate, the pattern shown in Fig. 6a transforms to that shown in Fig. 6b. Such a change of the image is explained as follows. When AC voltage is applied to the structure, the level of the depleted layer locates lower than microscratches (level 1, Fig. 7). When we additionally applied DC voltage, some negative ion charge (3, Fig. 7) accumulates near the silicon surface. It causes the increasing of current leakage $\mathbf{J}$ through nematic/silicon interface. Because of this the depleted layer width $\chi$ decreases. When $\chi$ becomes less than depth of microscratches (level 2, Fig. 7), the segments (S) of silicon surface can be considered as electrically isolated regions. When we illuminate silicon surface by a laser beam, within every such segments, different amounts of non-equilibrium carriers are generated due to the Gauss distribution of intensity of laser irradiation. Therefore, each segment will have different resistiv-

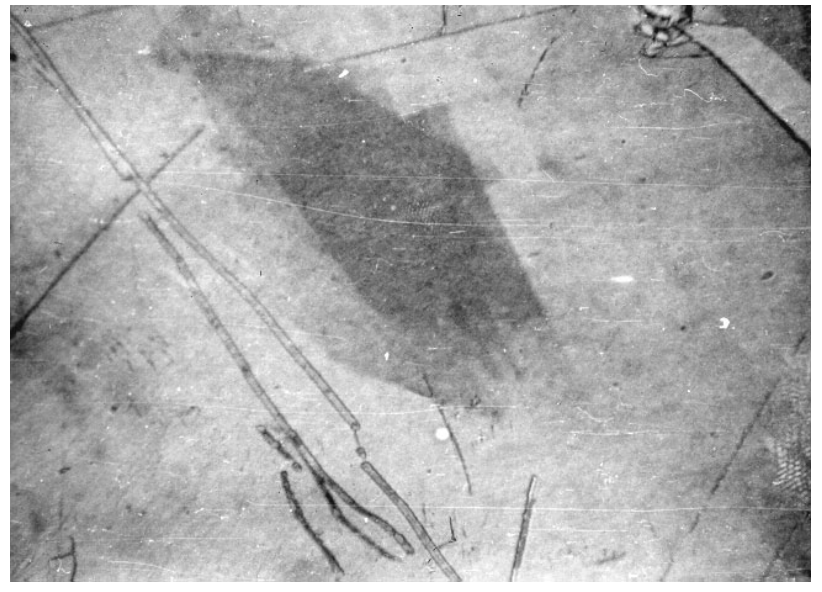

b

Fig. 6. Modulation of testing light by ITO/nematic/silicon/Al structure that is illuminated by diffracted He-Ne laser beam. $a$ - AC voltage $\left.\left(5.6 \mathrm{~V} ; 10^{4} \mathrm{~Hz}\right) . \mathrm{b}\right)$ the same AC voltage and DC voltage ("+" $2.5 \mathrm{~V}$ ) applied in addition. 


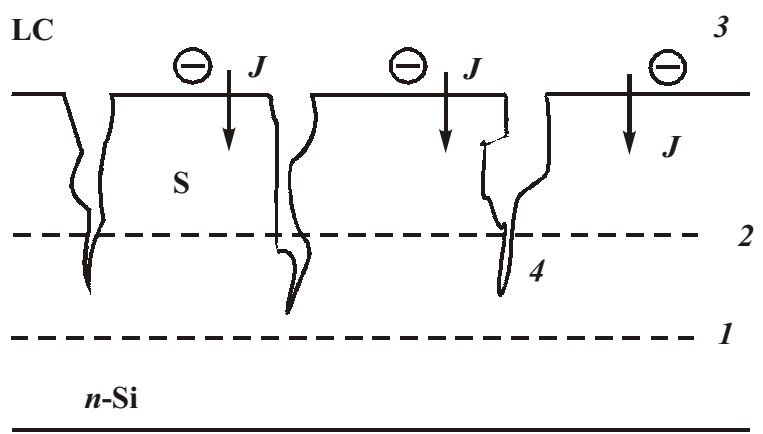

Al

Fig. 7. Schematic change of the depleted layer $\chi$ position in the silicon sample. $1-\chi$ level by AC voltage only, $2-\chi$ level by AC and additionally DC voltages action, 3 - negative charge, 4 microscratches of silicon surface.

ity and liquid crystal layer will "visualize" these segments of silicon surface separately.

\section{Conclusion}

The analysis of Freedericks transitions in the liquid crystal over different doping regions on silicon surface in ITO/nematic/silicon/Al structure, as well as photosensitivity of such a structure allow us to conclude that surface conductivity of silicon sample is modulated by surface localized ion charges. The investigated properties of long time transitions between states of the structure, which are due to an accumulated ion charge near the surface confirm that this charge is adsorbed at the silicon surface. The utilization of the ion induced field effect in such type of structures enables us: 1) to estimate the surface density of ion charges in liquid crystal; 2) to use such type of structures as spatial light modulator and 3) to test nondestructively the quality of silicon surface by changing the external voltage parameters.

\section{References}

1. R.Muller, T.Kamins, Device Electronics for Integrated Circuits, Wiley, 1986.

2. Gritsenko N.I., Kucheev S.I. "Field effect in silicon induced by charge in liquid crystal", Abstracts of $16^{\text {th }}$ Int. Liq. Crys. Conf. KENT(USA),-P.105(1996).

3. Gritsenko N.I., Kucheev S.I., "Electrooptic effects in liquid crystal for testing of silicon surface", Proc. of VIII th Int. Symp. of SID. Crimea (Ukraine), pp.235-238 (1999).

4. Gritsenko N.I., Kucheev S.I., "Domaine switch in siliconnematic-ITO structure" // Proc. of SPIE, 3488, pp.43-45 (1999).

5. Gritsenko N.I., Kucheev S.I., "Testing of silicon surface by nematic liquid crystals" // Microelectronics, 26, pp.341-343 (1997).

6. L. M. Blinov, Electrooptics and Magnetooptics of Liquid Crystals, Nauka, Moscow, 1977 (in Russian).

7. R. N. Thurston, J. Cheng, R. B. Meyer et all, "Physical mechanisms of de switching in a liquid crystal bistable boundary layer display" // J. Appl. Phys., 56, pp.263-281 (1984).

8. G.Barbero and G.Durand, "Selective ion absorption and nonlocal anchoring energy of nematic liquid crystals" // $J$. Appl. Phys., 67, pp.2678-2683 (1990).

9. G. Barbero, A.K. Zvezdin and Evangelista, "Ionic adsorption and equilibrium distribution of charges in a nematic cell" // Phys. Rev. E 59, pp.1846-1852 (1999).

10. V.Nazarenko, V.M.Pergamenshchick, O.V. Koval'chuk et all., "Non-Debay screening in nematic cell" // Phys. Rev. E 60, pp.5580-5587 (1999). 